\title{
RACSAM
}

Rev. R. Acad. Cien. Serie A. Mat.

VOL. 95 (1), 2001, pp. 1-6

Álgebra / Algebra

Comunicación Preliminar / Preliminary Communication

\section{Specializations of Jordan superalgebras}

\section{Martínez and E. Zelmanov}

\begin{abstract}
We construct universal associative enveloping algebras for a large class of Jordan superalgebras.

\section{Especializaciones de superálgebras de Jordan}

Resumen. Construimos álgebras envolventes universales asociativas para varias superálgebras de Jordan.
\end{abstract}

\section{Introduction}

Let $F$ be a ground field of characteristic $\neq 2$. A (linear) Jordan algebra is a vector space $J$ with a binary bilinear operation $(x, y) \rightarrow x y$ satisfying the following identities:

(J1) $x y=y x$

(J2) $\left(x^{2} y\right) x=x^{2}(y x)$

For an element $x \in J$ let $R(x)$ denote the right multiplication $R(x): a \rightarrow a x$ in $J$. If $x, y, z \in J$ then by $\{x, y, z\}$ we denote their Jordan triple product $\{x, y, z\}=(x y) z+x(y z)-y(x z)$.

A Jordan algebra $J$ is called special if it is embeddable into an algebra of type $A^{(+)}$, where $A$ is an associative algebra. The algebra $H_{3}(O)$ is exceptional. A homomorphism $J \rightarrow A^{(+)}$is called a specialization of a Jordan algebra $J$. N. Jacobson [3] introduced the notion of a universal associative enveloping algebra $U=U(J)$ of a Jordan algebra $J$ and showed that the category of specializations of $J$ is equivalent to the category of homomorphisms of the associative algebra $U(J)$.

Let $V$ be a Jordan bimodule over the algebra $J$ (see [3]). We call $V$ a one-sided bimodule if $\{J, V, J\}=$ (0). In this case, the mapping $a \rightarrow 2 R_{V}(a) \in E n d_{F} V$ is a specialization. The category of one-sided bimodules over $J$ is equivalent to the category of right (left) $U(J)$-modules.

N. Jacobson [3] found universal associative enveloping algebras for all simple finite dimensional Jordan algebras.

In this paper we study specializations and one-sided bimodules of Jordan superalgebras. Let us introduce the definitions.

By a superalgebra we mean a $Z / 2 Z$-graded algebra $A=A_{\overline{0}}+A_{\overline{1}}$. We define $|a|=0$ if $a \in A_{\overline{0}}$ and $|a|=1$ if $a \in A_{\overline{1}}$.

Presentado por Jesus Ildefonso Díaz.

Recibido: 8 de Septiembre 2001. Aceptado: 10 de Octubre 2001.

Palabras clave / Keywords: Specializations, Jordan superalgebras

Mathematics Subject Classifications: 17C70, 17C25

(C) 2001 Real Academia de Ciencias, España. 
For instance, if $V$ is a vector space of countable dimension, and $G(V)=G(V)_{\overline{0}}+G(V)_{\overline{1}}$ is the Grassmann algebra over $V$, that is, the quotient of the tensor algebra over the ideal generated by the symmetric tensors, then $G(V)$ is a superalgebra. Its even part is the linear span of all products of even length and the odd part is the linear span of all products of odd length.

If $A$ is a superalgebra, its Grassmann enveloping algebra is the subalgebra of $A \otimes G(V)$ given by $G(A)=A_{\overline{0}} \otimes G(V)_{\overline{0}}+A_{\overline{1}} \otimes G(V)_{\overline{1}}$.

Let $\mathcal{V}$ be a homogeneous variety of algebras, that is, a class of $F$-algebras satisfying a certain set of homogeneous identities and all their partial linearizations (see [20]).

Definition 1 A superalgebra $A=A_{\overline{0}}+A_{\overline{1}}$ is called a $\mathcal{V}$ superalgebra if $G(A) \in \mathcal{V}$.

C. T. C. Wall [19] showed that every simple finite-dimensional associative superalgebra over an algebraically closed field $F$ is isomorphic to the superalgebra

$M_{m, n}(F)=\left\{\left(\begin{array}{cc}A & 0 \\ 0 & D\end{array}\right), A \in M_{m}(F), D \in M_{n}(F)\right\}+\left\{\left(\begin{array}{cc}0 & B \\ C & 0\end{array}\right), B \in M_{m \times n}(F), C \in M_{n \times m}(F)\right\}$

or to the superalgebra

$$
P(n)=\left\{\left(\begin{array}{cc}
A & 0 \\
0 & A
\end{array}\right), A \in M_{n}(F)\right\}+\left\{\left(\begin{array}{cc}
0 & B \\
B & 0
\end{array}\right), B \in M_{n}(F)\right\} .
$$

Jordan superalgebras were first studied by V. Kac [5] and I. Kaplansky [10,11]. In [5] V. Kac (see also I. L. Kantor [8,9]) classified simple finite dimensional Jordan superalgebras over an algebraically closed field of zero characteristic. In [16] this classification was extended to simple finite dimensional Jordan superalgebras, with semisimple even part, over characteristic $p>2$; a few new exceptional superalgebras in characteristic 3 were added to the list. In [13] the remaining case of Jordan superalgebras with nonsemisimple even part was tackled.

Let's consider the examples that arise in these classifications.

If $A=A_{\overline{0}}+A_{\overline{1}}$ is an associative superalgebra then the superalgebra $A^{(+)}$, with the new product $a \cdot b=\frac{1}{2}\left(a b+(-1)^{|a||b|} b a\right)$ is Jordan. This leads to two superalgebras:

1) $M_{m, n}^{(+)}(F), m \geq 1, n \geq 1$;

2) $P(n)^{(+)}, n \geq 2$;

If $A$ is an associative superalgebra and $\star: A \rightarrow A$ is a superinvolution, that is, $\left(a^{\star}\right)^{\star}=a,(a b)^{\star}=$ $(-1)^{|a||b|} b^{\star} a^{\star}$, then $H(A, \star)=H\left(A_{\overline{0}}, \star\right)+H\left(A_{\overline{1}}, \star\right)$ is a subsuperalgebra of $A^{(+)}$. The following two subalgebras of $M_{m, n}^{(+)}$are of this type:

3) $O s p_{m, n}(F)$ if $n=2 k$ is even. The superalgebra consists of matrices $\left(\begin{array}{ll}A & B \\ C & D\end{array}\right)$, where $A^{t}=A \in$ $M_{m}(F), C=J^{-1} B^{t} \in M_{n \times m}(F), D=J^{-1} D^{t} J \in M_{n}(F), J=\left(\begin{array}{cc}0 & I_{k} \\ -I_{k} & 0\end{array}\right) ;$

4) $Q(n)=\left\{\left(\begin{array}{ll}A & B \\ C & D\end{array}\right), D=A^{t}, B^{t}=B, C^{t}=-C \in M_{n}(F)\right\}$.

5) Let $V=V_{\overline{0}}+V_{\overline{1}}$ be a $Z / 2 Z$-graded vector space with a superform $<,>: V \times V \rightarrow F$ which is symmetric on $V_{\overline{0}}$, skewsymmetric in $V_{\overline{1}}$ and $<V_{\overline{0}}, V_{\overline{1}}>=(0)=<V_{\overline{1}}, V_{\overline{0}}>$.

The superalgebra $J=F 1+V=\left(F 1+V_{\overline{0}}\right)+V_{\overline{1}}$ is Jordan. 
6) The 3-dimensional Kaplansky superalgebra, $K_{3}=F e+(F x+F y)$, with the multiplication $e^{2}=$ $e, \quad e x=\frac{1}{2} x, e y=\frac{1}{2} y, \quad[x, y]=e$.

7) The 1-parametric family of 4-dimensional superalgebras $D_{t}$ is defined as $D_{t}=\left(F e_{1}+F e_{2}\right)+(F x+$ $F y)$ with the product: $e_{i}^{2}=e_{i}, e_{1} e_{2}=0, e_{i} x=\frac{1}{2} x, e_{i} y=\frac{1}{2} y, x y=e_{1}+t e_{2}, i=1,2$.

The superalgebra $D_{t}$ is simple if $t \neq 0$. In the case $t=-1$, the superalgebra $D_{-1}$ is isomorphic to $M_{1,1}(F)$.

8) The 10-dimensional Kac superalgebra (see [5]) has been proved to be exceptional in [15]. In characteristic 3 this superalgebra is not simple, but it has a subalgebra of dimension 9 that is simple (the degenerated Kac superalgebra. There are two other examples of simple Jordan superalgebras in ch $\mathrm{F}=3$, both of them exceptional (see [16]).

9) We will consider now Jordan superalgebras defined by a bracket.

If $A=A_{\overline{0}}+A_{\overline{1}}$ is an associative commutative superalgebra wit a bracket on $A,\{\}:, A \times A \rightarrow A$, the Kantor Double of $(A,\{\}$,$) is a the superalgebra J=A+A x$ with the $Z / 2 Z$ gradation $J_{\overline{0}}=A_{\overline{0}}+$ $A_{\overline{1}} x, J_{\overline{1}}=A_{\overline{1}}+A_{\overline{0}} x$ and the multiplication in $J$ given by: $a(b x)=(a b) x, \quad(b x) a=(-1)^{|a|}(b a) x$, $(a x)(b x)=(-1)^{|b|}\{a, b\}$, and the product (in $J$ ) of two elements of $A$ is just the product of them in $A$.

A bracket on $A$ is called a Jordan bracket if the Kantor Double $J(A,\{\}$,$) is a Jordan superalgebra.$ Every Poisson bracket is a Jordan bracket.

10) Let $Z$ be a unital associative commutative algebra with a derivation $d: Z \rightarrow Z$. Consider the superalgebra $C K(Z, d)=A+M$, where $A=J_{\overline{0}}=Z+\sum_{i=1}^{3} w_{i} Z, M=J_{\overline{1}}=x Z+\sum_{i=1}^{3} x_{i} Z$ are free $Z$-modules of rank 4. The multiplication on $A$ is $Z$-linear and $w_{i} w_{j}=0, i \neq j, w_{1}^{2}=w_{2}^{2}=1, w_{3}^{2}=-1$.

Denote $x_{i \times i}=0, \quad x_{1 \times 2}=-x_{2 \times 1}=x_{3} \quad x_{1 \times 3}=-x_{3 \times 1}=x_{2}, \quad-x_{2 \times 3}=x_{3 \times 2}=x_{1}$.

The bimodule structure and the bracket on $M$ are defined via the following tables:

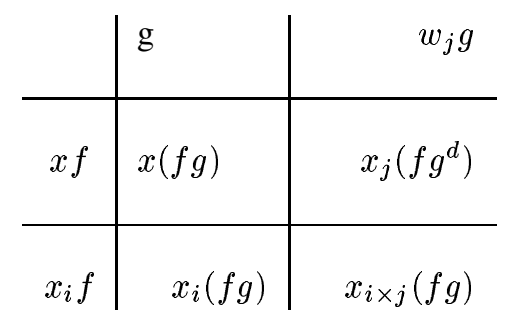

\begin{tabular}{c|c|c} 
& $x g$ & $x_{j} g$ \\
\hline$x f$ & $f^{d} g-f g^{d}$ & $-w_{j}(f g)$ \\
\hline$x_{i} f$ & $w_{i}(f g)$ & 0
\end{tabular}

The superalgebra $C K(Z, d)$ is simple if and only if $Z$ does not contain proper d-invariant ideals.

In [5], [8] it was shown that simple finite dimensional Jordan superalgebras over an algebraically closed field $F$ of zero characteristic are those of examples 1) - 8) and the Kantor Double (example 9) of the Grassmann algebra with the bracket $\{f, g\}=\sum(-1)^{|f|} \frac{\partial f}{\partial \xi_{i}} \frac{\partial g}{\partial \xi_{i}}$.

The examples 9), 10) are related to infinite dimensional superconformal Lie superalgebras (see [6], [7]). In particular, the superalgebras $C K(Z, D)$ correspond to an important superconformal algebra discovered in [1] and [2].

In [13] it was shown that the only simple finite dimensional Jordan superalgebras over an algebraically closed field of characteristic $p>2$ with nonsemisimple even part are superalgebras 9),10) built on truncated polynomials. 


\section{Universal enveloping algebras}

In what follows the ground field $F$ is assumed to be algebraically closed.

1. Let $U$ be a universal associative enveloping algebra of a special Jordan superalgebra $J, u: J \rightarrow U$ a universal specialization. The algebra $U$ is equipped with a natural superinvolution $\star$ leaving all elements from $u(J)$ fixed. Then $u(J) \subseteq H(U, \star)$. We call a superalgebra $J$ reflexive if $u(J)=H(U, \star)$.

Theorem 1 All superalgebras of examples 1) - 4) are reflexive except the following ones: $M_{1,1}^{(+)}(F)$, $O s p(1,2) \simeq D(-2), Q(2)$. Hence,

$$
\left\{\begin{array}{l}
U\left(M_{m, n}^{(+)}(F)\right) \simeq M_{m, n}(F) \oplus M_{m, n}(F) \quad \text { for }(m, n) \neq(1,1) \\
U\left(P^{(+)}(n)\right)=P(n) \oplus P(n), \quad n \geq 2 ; \\
U(o s p(m, n)) \simeq M_{m, n}(F), \quad(m, n) \neq(1,2) ; \\
U(Q(n)) \simeq M_{n, n}(F), \quad n \geq 3 .
\end{array}\right.
$$

2. Let $Z$ be an associative commutative algebra with a derivation $D: Z \rightarrow Z$. Let $W=<Z, D>$ and let $u: C K(Z, D) \rightarrow \quad M_{2,2}(W)$ be the embedding found in [12]

The embedding $u$ extends the embedding of Kantor doubles of brackets of vector type found in [14]

Theorem $2 U(C K(Z, D))=M_{2,2}(W)$, the embedding $u$ is universal.

3. The superalgebra of $C K(Z, D)$ spanned over $F$ by the elements $1, w_{1}, w_{2}, w_{3}, x, x_{1}, x_{2}, x_{3}$ is isomorphic to $Q(2)$.

Theorem 3 The restriction of the embedding $u$ (see above) to $Q(2)$ is a universal specialization;

$$
U(Q(2)) \simeq M_{2,2}(F[t])
$$

where $F[t]$ is a polynomial algebra in one variable.

4. Let us describe the universal associative enveloping superalgebra of $M_{1,1}(F)$. Consider the ring of polynomials and the field of rational functions in two variables, $F\left[z_{1}, z_{2}\right] \subseteq F\left(z_{1}, z_{2}\right)$. Let $K$ be the quadratic extension of $F\left(z_{1}, z_{2}\right)$ generated by a root of the equation $a^{2}+a-z_{1} z_{2}=0$. Consider the subring $A=F\left[z_{1}, z_{2}\right]+F\left[z_{1}, z_{2}\right] a$ and the subspaces $M_{12}=F\left[z_{1}, z_{2}\right]+F\left[z_{1}, z_{2}\right] a^{-1} z_{2}, M_{21}=$ $F\left[z_{1}, z_{2}\right] z_{1}+F\left[z_{1}, z_{2}\right] a$ of $K$. Then $U=\left(\begin{array}{cc}A & M_{12} \\ M_{21} & A\end{array}\right)$ is a subring of $M_{2}(K)$.

Theorem $4 U\left(M_{1,1}(F)\right) \simeq\left(\begin{array}{cc}A & M_{12} \\ M_{21} & A\end{array}\right)$. The mapping

$$
u:\left(\begin{array}{ll}
\alpha_{11} & \alpha_{12} \\
\alpha_{21} & \alpha_{22}
\end{array}\right) \rightarrow\left(\begin{array}{cc}
\alpha_{11} & \alpha_{12}+\alpha_{21} a^{-1} z_{2} \\
\alpha_{12} z_{1}+\alpha_{21} a & \alpha_{22}
\end{array}\right)
$$

is a universal specialization.

5. Let $V=V_{\overline{0}}+V_{\overline{1}}$ be a $Z / 2 Z$-graded vector space, $\operatorname{dim} V_{\overline{0}}=m, \operatorname{dim} V_{\overline{1}}=2 m$; let $<,>: V \times V \rightarrow F$ be a supersymmetric bilinear form on $V$. The universal associative enveloping algebra of the Jordan algebra $F 1+V_{\overline{0}}$ is the Clifford algebra $C l(m)=\left\langle 1, e_{1}, \ldots, e_{m} \mid e_{i} e_{j}+e_{j} e_{i}=0, i \neq j, e_{i}^{2}=1\right\rangle$ (see [3]). Assuming the generators $e_{1}, \ldots, e_{m}$ to be odd, we get a $Z / 2 Z$-gradation on $C L(m)$.

In $V_{\overline{1}}$ we can find a basis $v_{1}, w_{1}, \ldots, v_{n}, w_{n}$ such that $\left.\left\langle v_{i}, w_{j}\right\rangle=\delta_{i j},\left\langle v_{i}, v_{j}\right\rangle=<w_{i}, w_{j}\right\rangle=0$. Consider the Weyl algebra $W_{n}=<1, x_{i}, y_{i}, 1 \leq i \leq n \mid\left[x_{i}, y_{j}\right]=\delta_{i j},\left[x_{i}, x_{j}\right]=\left[y_{i}, y_{j}\right]=0>$. Assuming 
$x_{i}, y_{i}, 1 \leq i \leq n$ to be odd, we make $W_{n}$ a superalgebra. The universal associative enveloping algebra of $F 1+V$ is isomorphic to the (super)tensor product $C l(m) \otimes_{F} W_{n}$.

6. Let $\operatorname{osp}(1,2)$ denote the Lie subsuperalgebra of $M_{1,2}(F)$ which consists of skewsymmetric elements with respect to the orthosympletic superinvolution. Let $x, y$ be the standard basis of the odd part of $\operatorname{osp}(1,2)$.

Theorem 5 (I. Shestakov) The universal enveloping algebra of $K_{3}$ is isomorphic to $U\left(\right.$ osp $(1,2) / i d\left([x, y]^{2}\right.$ $-[x, y]))$, where $U(\operatorname{osp}(1,2))$ is the universal associative enveloping algebra of osp $(1,2)$ and $i d\left([x, y]^{2}-\right.$ $[x, y]))$ is the ideal of $U(\operatorname{osp}(1,2))$ generated by $[x, y]^{2}-[x, y]$.

Clearly, if $\operatorname{ch} F=0$ then $K_{3}$ does not have nonzero specializations that are finite dimensional algebras. If $\operatorname{ch} F=p>0$ then $K_{3}$ has such specializations.

7. Let us consider the superalgebras $D(t)$. We will assume that $t \neq-1,0,1$, because $D(-1) \simeq$ $M_{1,1}(F) ; D(0) \simeq K_{3}+F ; D(1)$ is a Jordan superalgebra of a superform.

Theorem 6 (I. Shestakov) The universal enveloping algebra of $D(t)$ is isomorphic to

$$
U\left(\operatorname{osp}(1,2) / i d\left([x, y]^{2}-(1+t)[x, y]+t\right)\right.
$$

Corollary 1 If chF $=0$ then all finite dimensional one-sided bimodules over $D(t)$ are completely reducible.

Indeed, it is known (see [4]) that finite dimensional representations of the Lie superalgebra $\operatorname{osp}(1,2)$ are completely reducible.

Now we will assume thar $\operatorname{ch} F=0$ and will classify irreducible finite dimensional one-sided bimodules over $D(t)$. Let us first consider four infinite dimensional Verma type right modules over $U(D(t)$. Each of these bimodules is generated by an even highest weight element $v$.

$V_{1}(t)=v U(d(t))$. Defining relations: $v(x y+y x)=(2 t+1) v, v y^{2}=0, v e_{1}=v, v e_{2}=0$. Basis: $v, v y, v x^{i}, i \geq 1$.

$V_{2}(t)=v U(d(t))$. Defining relations: $v(x y+y x)=(2 t+1) v, v y=0, v e_{1}=v, v e_{2}=0$. Basis: $v, v x^{i}, i \geq 1$.

Changing parity we get two new bimodules $V_{1}(t)^{o p}$ and $V_{2}(t)^{o p}$.

Each of these bimodules has the unique irreducible homomorphism image $W_{1}(t)$ or $W_{2}(t)$ or $W_{1}(t)^{o p}$ or $W_{2}(t)^{o p}$ respectively.

Theorem 7 If $t=\frac{-(m+1)}{m}, m \geq 1$, then $D(t)$ has two irreducible finite dimensional one sided bimodules $W_{1}(t)$ and $W_{1}(t)^{o p}$.

If $t=\frac{-m}{m+1}, m \geq 1$, then $D(t)$ has two irreducible finite dimensional one sided bimodules $W_{2}(t)$ and $W_{2}(t)^{o p}$.

If $t$ can not be represented as $-\frac{m+1}{m}$ or $\frac{-m}{m+1}$, where $m$ is a positive integer, then $D(t)$ does not have nonzero finite dimensional specializations.

If $\operatorname{ch} F=p>2$ then for an arbitrary $t$ the superalgebra $D(t)$ can be embedded into a finite dimensional associative superalgebra. It suffices to notice that $D(t) \subseteq C K\left(F\left[t \mid t^{p}=0\right], d / d t\right)$. 


\section{References}

[1] Cheng, S. J. and Kac, V. G. (1997). A New N=6 superconformal algebra, Comm. Math. Phys. 186.

[2] Grozman, P., Leites, D. and Shchepochkina, I., Lie superalgebras of string theories, hep-th 9702120.

[3] Jacobson, N. (1969). Structure and Representation of Jordan algebras, Amer. Math. Soc. Providence, R.I.

[4] Kac, V. G. (1977). Lie Superalgebras, Adv. Math. 26, 8-96.

[5] Kac, V. G. (1977). Classification of simple Z-graded Lie superalgebras and simple Jordan superalgebras, Comm. Algebra 5 (13), 1375-1400.

[6] Kac, V. G. and van de Leur, J. W. (1989). On classification of superconformal algebras, Strings 88, World Scientific, Singapore, 77-106.

[7] Kac, V. G., Martínez, C. and Zelmanov, E. (2001). Graded simple Jordan superalgebras of growth one, Mem. Amer. Math. Soc. 150, 140pp.

[8] Kantor, I. L. (1989). Connections between Poisson brackets and Jordan and Lie superalgebras, Lie theory, differential equations and representation theory, 213-225, Montreal.

[9] Kantor, I. L. (1992). Jordan and Lie superalgebras defined by Poisson brackets, Algebra and Analysis, 55-79, 1989. Amer. Math. Soc. Transl. Ser. (2), 151.

[10] Kaplansky, I. (1980). Superalgebras, Pacific J. Math. 86, 93-98.

[11] Kaplansky, I., Graded Jordan Algebras I, Preprint.

[12] Martínez, C., Shestakov, I. P. and Zelmanov, E. (2001). Jordan algebras defined by brackets, To appear in J. London Math. Soc.

[13] Martínez, C. and Zelmanov, E. (2001). Simple finite dimensional Jordan superalgebras of prime characteristic, $J$. Algebra 236 no.2, 575-629.

[14] McCrimmon, K. (1992). Speciality and nonspeciality of two Jordan superalgebras, J. Algebra 149, 326-351.

[15] Medvedev, Y. and Zelmanov, E. (1992). Some counterexamples in the theory of Jordan Algebras, Nonassociative Algebraic Models, Nova Science Publish., S. González and H.C. Myung eds., 1-16.

[16] Racine, M. and Zelmanov, E. (2001). Classification of simple Jordan superalgebras with semisimple even part, to appear.

[17] Shestakov, I.(2000). Universal enveloping algebras of some Jordan superalgebras, personal communication.

[18] Shtern, A. S. (1987). Representation of an exceptional Jordan superalgebra, Funktsional Anal. i Prilozhen 21, 93-94.

[19] Wall, C. T. C. (1964). Graded Brauer groups, textit J. Reine Angew. Math. 213, 187-199.

[20] Zhevlakov K. A., Slinko, A. M., Shestakov, I. P. and Shirshov, A. I., Rings that are rearly associative, Academic Press, New York.

$\begin{array}{ll}\text { C. Martínez } & \text { E. Zelmanov } \\ \text { Departamento de Matemáticas } & \text { Department of Mathematics } \\ \text { Universidad de Oviedo } & \text { Yale University } \\ \text { 33007 Oviedo, Spain } & \text { New Haven, CT 06520, USA }\end{array}$

